will provide troop protection and lead to increased security. Considering that Afghanistan is facing a steady deterioration in security with increases in both insurgent activity and crime, it might be time to revise this presumption. Moreover, recent research into community perceptions of PRTs and their work indicates that Afghans do not want military forces to carry out development and reconstruction projects because they feel there are "strings attached" to such projects and because the accountability of military forces is low: at the same time Afghans do see a role for the international military forces in security sector reform. ${ }^{21}$ Military forces should focus on security tasks and security sector reform, rather than continue their efforts in reconstruction and development where there are other actors with greater expertise.

\section{Conclusion}

Over the past years NATO has shown an increased interest in hearing NGO perspectives. NGO representatives are invited to meet with incoming members of the PRT, they are asked

21 Azarbaijani-Moghaddam and Wardak (2008). to speak at the NATO school and participate in international seminars and conferences. Although opportunities for public and constructive discussions are very much appreciated by the NGO sector, it has not necessarily led to noticeable changes on the ground.

Afghanistan represents an incredibly challenging scenario for NGOs. In the North NGOs often see themselves as partners in the peacebuilding strategy of the international mission while in the South NGOs are unwilling to be partners in the parallel warfighting strategy.

NGOs have had to reconcile their commitment to support needy Afghan communities with an operating environment created by international strategies they often directly oppose. No one suggests that NGOs have all the answers. However, for the best possible strategies for Afghan recovery to emerge, the views of the NGO sector and, those of the communities they work with, must be more given more than a token role in the policy dialogue with NATO. This dialogue needs to focus on other pressing issues beyond the militarization of aid, such as increasing insecurity, the predicted humanitarian crisis caused by continuing drought and food prices, and aid effectiveness for Afghanistan.

\title{
„Counterinsurgency“ - Neue Einsatzformen für die NATO?
}

\author{
Jochen Hippler*
}

\begin{abstract}
NATO has shifted its operational focus without a clear strategy. The Strategic Concept of 1999 is vague and leaves open a wide range of options. The US military has further developed earlier concepts of counterinsurgency. Boosted by what is seen as a successful application in Iraq, counterinsurgency is also being promoted in Afghanistan. However, there are major difficulties and deficits in applying counterinsurgency strategy, particularly in Afghanistan. Instead of adopting a counterinsurgency strategy for Afghanistan, NATO members should develop alternative strategies with a focus on political and economic reforms.
\end{abstract}

Keywords: NATO, military strategy, U.S., Afghanistan, Iraq; NATO, Militärstrategie, USA, Afghanistan, Irak

\section{Einleitung}

Afghanistan stellt eine besondere und qualitativ neue Herausforderung für die NATO dar. Die konventionelle und atomare Rüstung der NATO-Mitgliedsländer und ihre überwältigende militärische Überlegenheit sind offensichtlich keine Garantie dafür, die Aufgabe der Stabilisierung und des State-Building in Afghanistan erfolgreich zu bewältigen. Im Gegenteil: Seit 2004/2005 hat sich die Sicherheitslage dramatisch zugespitzt und die politische Situation zu einer kaum verhüllten Krise ent-

\footnotetext{
* PD Dr. Jochen Hippler ist wissenschaftlicher Mitarbeiter am Institut für Entwicklung und Frieden an der Universität Duisburg.
}

wickelt. Die Geheimverhandlungen mit den Taliban sind ein Ausdruck dieser Situation. Ohne die Erkenntnis, in Afghanistan in eine Sackgasse geraten zu sein, wäre die Unterstützung dieser Gespräche durch die NATO-Führungsmächte unvorstellbar. Die Erklärung für die dürftige Bilanz des NATO-Einsatzes in Afghanistan trotz der überwältigenden militärischen Überlegenheit des Bündnisses über die Aufständischen liegt in der Tatsache, dass der Afghanistankrieg keine konventionelle militärische Auseinandersetzung darstellt, sondern vor allem in Aufstandsbekämpfung besteht. Und diese Einsatzform - Counterinsurgen$c y$ - folgt ganz anderen Regeln als ein regulärer Krieg. Und auf sie sind die NATO und ihre Mitgliedsländer kaum vorbereitet, 
da dies ihren Erfahrungen und Traditionen grundlegend widerspricht.

\section{Wandel der Einsatzformen}

Die Zeiten, in denen sich die NATO im Rahmen des Ost-WestKonflikts vor allem auf Europa konzentrierte und dabei weniger auf tatsächliche Kriegführung, sondern auf „Abschreckung“ setzte, sind lange vorbei. Seit dem Ende des Kalten Krieges werden regionalen Gewaltkonflikten durch die westlichen Regierungen und die NATO ein weit größeres Gewicht zugemessen; Abschreckung, Landes- und Bündnisverteidigung haben stark an Bedeutung verloren. Selbst die Bundeswehr wurde von einer Abschreckungstruppe zu einer „Armee im Einsatz“. Westliches Militär wird leichter, schneller und häufiger eingesetzt als während des Kalten Krieges, wobei die offiziell vorgebrachten Begründungen („neue Bedrohungen“, „humanitäre Erfordernisse“) weniger ins Gewicht fallen dürften als die Tatsache, dass die Hemmschwelle einer Gewaltanwendung niedriger liegt, weil kaum noch Eskalationsgefahr auf eine strategische Ebene besteht - schließlich ist es ja nicht so, dass es ethnische Konflikte und humanitäre Notlagen früher nicht gegeben hätte. Allerdings reagiert man heute anders darauf.

Diese Veränderung schlug sich in neuen Einsatz- und Operationsformen der Bündnispartner und der NATO selbst nieder, aber auch in der gegenüber der Fassung von 1991 deutlich veränderten NATO-Strategie vom April 1999 („Das Strategische Konzept des Bündnisses“, im Folgenden zitiert nach der amtlichen deutschen Fassung $)^{1}$. In Abschnitt 20 der Strategie wird formuliert: „Die Sicherheit des Bündnisses bleibt einem breiten Spektrum militärischer und nichtmilitärischer Risiken unterworfen, die aus vielen Richtungen kommen und oft schwer vorherzusagen sind. $\mathrm{Zu}$ diesen Risiken gehören Ungewißheit und Instabilität im und um den euro-atlantischen Raum sowie die mögliche Entstehung regionaler Krisen an der Peripherie des Bündnisses, die sich rasch entwickeln könnten. Einige Länder im und um den euro-atlantischen Raum sehen sich ernsten wirtschaftlichen, sozialen und politischen Schwierigkeiten gegenüber. Ethnische und religiöse Rivalitäten, Gebietsstreitigkeiten, unzureichende oder fehlgeschlagene Reformbemühungen, die Verletzung von Menschenrechten und die Auflösung von Staaten können zu lokaler und selbst regionaler Instabilität führen. Die daraus resultierenden Spannungen könnten zu Krisen führen, die die euro-atlantische Stabilität berühren, sowie zu menschlichem Leid und bewaffneten Konflikten. Solche Konflikte könnten, indem sie auf benachbarte Staaten einschließlich NATO-Staaten übergreifen oder in anderer Weise, auch die Sicherheit des Bündnisses oder anderer Staaten berühren." (alle Hervorhebungen durch den Autor)

Euphemistisch lässt sich formulieren, dass diese Vorstellung von Sicherheitspolitik höchst flexibel ist, sich auf nichts Substanzielles festlegt und zugleich alle Möglichkeiten der Militär- und Sicherheitspolitik out-of-area offen lässt. Es ließe sich aber auch feststellen, dass solche Programmatik in höchstem Maß vage und unklar bleibt. Letztlich wird hier eine Allzuständigkeit für die Bewältigung auch unklarer, noch unbekannter

1 NATO, Das Strategische Konzept des Bündnisses April 1999, Webseite der deutschen Vertretung bei der NATO. und auch „schwer vorherzusagender“ Risiken reklamiert. Auch mandatiert sich die NATO hier selbst zur Bearbeitung all dessen, was die „Stabilität“ bedroht (ein höchst bedeutungsoffener Begriff) und „die Sicherheit des Bündnisses oder anderer Staaten berühren " könnte. Wenn aber der Sicherheitsbegriff vorher so weit ausgedehnt wird, gibt es kaum noch Entwicklungen, die sie nicht bedrohen „könnten“. Überspitzt formuliert: Die NATO verpflichtet sich hier zu nichts, ermächtigt sich aber zu allem - je nach aktueller Einschätzung der Situation. Selbst das „organisierte Verbrechen“ die „Unterbrechung der Zufuhr lebenswichtiger Ressourcen“ oder „die unkontrollierte Bewegung einer großen Zahl von Menschen“ werden in die Zuständigkeit des Bündnisses gestellt.

Sehen wir uns einige der militärischen Einsätze und deren Kontexte der letzten Jahre an. Die wenigsten waren offizielle NATO-Einsätze (wie heute der Afghanistan-Einsatz), aber führende NATO-Mitglieder waren uni- oder multilateral daran beteiligt und nutzten dabei durchaus auch Infrastruktur, die der NATO zuzurechnen ist. Der Irak (1991), Somalia, Bosnien, Kosovo stellten den Beginn solcher Einsätze dar, Afghanistan und erneut der Irak (2003) bilden aktuelle Fälle. Zahlreiche andere Operationen blieben von beschränkterem Umfang. Es ist deutlich, dass die Gesamtheit solcher Einsätze unter höchst unterschiedlichen politischen, rechtlichen und institutionellen Rahmenbedingungen erfolgte, dass sie aber häufig aus zwei Komponenten bestanden: einmal konventionellen militärischen Einsatzformen, einschließlich der Anwendung militärischer Gewalt (Luftangriffe, bewaffnete Kampfeinsätze am Boden, etc.) gegen bewaffnete feindliche Kräfte staatlicher oder substaatlicher Akteure.

Zugleich und häufig daran anschließend kam es nicht selten zu komplexeren Militäreinsätzen, die nicht primär auf die Zerschlagung eines militärischen Gegners zielten, sondern auf die Gewährleistung von Stabilität und Sicherheit in einem Zielland, auf die Leistung infrastruktureller oder humanitärer Hilfe, die Flankierung politischer Aufbau- oder Umgestaltungsprozesse (z.B. im Kontext von Nation-Building oder State-Building) oder ähnliches. Das gehört in der US-Militärpolitik zur Kategorie der „Military Operations Other than War“. Hierbei wurden dem Militär häufig politische oder Ordnungsfunktionen übertragen, die administrative, quasi-polizeiliche, infrastrukturelle oder eben politische Komponenten ins Zentrum rückten, während die klassischen militärischen Funktionen des Militärs (Anwendung von Gewalt, Kampfeinsätze) eher Hilfsaufgaben erfüllten oder als Drohpotenzial im Hintergrund blieben. Diese Situation entstand in zahlreichen, durchaus unterschiedlichen Kontexten, etwa im Rahmen humanitärer Hilfeleistung durch Militär, in Post-Conflict-Situationen, bei fragiler oder gescheiterter Staatlichkeit und beim intendierten oder naturwüchsigen Nation-Building. Soldaten von NATO-Mitgliedsländern, und in wichtigen Einzelfällen auch die NATO insgesamt, gerieten so in oft ungewohnte, von ihnen nicht selten als kompliziert oder unangenehm empfundene Situationen, für die sie oft nicht vorbereitet oder ausgebildet waren.

Solche unkonventionellen Einsatzformen "Other than War“ (bzw. Mischformen dieser mit konventionellen Elementen) nahmen und nehmen an Zahl und Bedeutung zu. Sie sind von der nicht mehr ganz neuen NATO-Strategie durchaus gedeckt 
- da diese in ihrer umfassenden Unbestimmtheit kaum etwas ausschließt. Allerdings entstehen dabei einige Probleme bzw. offene Fragen: a) Die NATO insgesamt verfügt für solche Einsatzformen trotz ihrer breiten Zielformulierung bisher über kein gemeinsames und abgestimmtes Konzept, weder auf der strategischen noch auf der taktischen Ebene; b) es gibt keine klaren Kriterien, nach denen über solche Einsätze entschieden würde, sondern eher freihändige Ad-hoc-Entscheidungen nach politischer Opportunität; c) einige NATO-Mitglieder (insbesondere die USA und die ehemaligen Kolonialmächte) verfügen auf dem Gebiet solcher unkonventioneller Militäreinsätze über beträchtliche Erfahrung, Konzepte und Ausbildung, während dies für andere Mitgliedsländer gar nicht oder nur sehr eingeschränkt zutrifft - etwa auch für die Bundeswehr. Dadurch können nicht nur Probleme vor Ort entstehen, sondern auch eine Schieflage bei der Entscheidung und Rahmensetzung über solche Operationen innerhalb des Bündnisses oder bei multilateralen Einsätzen.

\section{US-amerikanische Einsatzkonzepte im Wandel}

Vor diesem Hintergrund ist es sinnvoll, sich entsprechende militärische Konzepte der darin erfahreneren Bündnispartner einmal anzusehen, da diese sich mangels fundierter Alternativen mit hoher Wahrscheinlichkeit ohnehin schleichend auch bei anderen durchsetzen dürften. Das gilt insbesondere für Konzepte der NATO-Führungsmacht USA.

Im US-amerikanischen Militär (insbesondere in der US-Army und dem Marine-Corps) wird seit Jahrzehnten an Fragen militärischer Einsätze unterhalb der Kriegsschwelle konzeptionell gearbeitet, auch wenn die Begrifflichkeiten gewissen Moden unterliegen und sich immer wieder änderten. Sprach man früher von „Small Wars“, in den 1960er Jahren vor allem von Aufstandsbekämpfung („Counterinsurgency“) und unter Präsident Reagan von „low-intensity conflict“ (LIC; auch: lowintensity warfare), so ist heute - wie erwähnt - von „Military Operations Other than War“ (MOOTW) und "Stability and Support Operations“ die Rede. ${ }^{2}$ In der aktuellen Variante, die seit Anfang/Mitte der 1990er Jahre gilt, zählen u.a. folgende Typen von Einsätzen dazu: Terrorismusbekämpfung, Bekämpfung von Drogenhandel und -produktion (Counterdrug Operations), die Durchsetzung von Sanktionen und Schutzzonen, die Sicherung von Luft- und Seeverkehrswegen, humanitäre Hilfe, militärische Unterstützung ziviler Behörden, Aufstandsbekämpfung, Unterstützung von Aufständen, Evakuierungsoperationen, Friedenseinsätze, Rettungs- und Bergungseinsätze, militärische Machtdemonstrationen (Show of Force Operations), punktuelle Angriffe (Strikes and Raids).

Insgesamt wird deutlich, dass viele dieser Aktivitäten auf die Umsetzung dessen zielen, was später in die NATO-Strategie aufgenommen wurde. Alle diese Operationsarten bestehen jeweils wieder aus verschiedenen Untertypen. Seit fast 30 Jahren gibt es dazu in der militärischen Community der USA eine lebhafte De-

2 Siehe ausführlich: Jochen Hippler, Counterinsurgency and Political Control US Military Strategies Regarding Regional Conflict, INEF-Report No. 81/2006, Institute for Development and Peace (INEF), University of Duisburg-Essen 2006; im Internet u.a. unter: www.jochen-hippler.de/neu/pdf-Dokumente/ INEF-Rep81.pdf. batte, die sich in der kontinuierlichen Weiterentwicklung militärischer Doktrinbildung niedergeschlagen hat. Die US-Army, das Marine Corps, die Joint Chiefs of Staff und - in geringerem Maße - die US Navy haben seitdem verbindliche Konzeptionsund Planungspapiere und Handbücher erarbeitet. Bereits diese erste Aufzählung der Einsatztypen lässt erkennen, dass die breite Aufgabenstellung der NATO-Strategie in den USA auf einen konzeptionellen Unterbau trifft, über den die NATO selbst und viele Mitgliedsländer nicht verfügen. Viele der Unterkonzepte und ihre Elemente erscheinen auf den ersten Blick unschuldig genug, teilweise verbergen sich hinter ihnen aber doch zahlreiche Punkte, die Anlass zu Diskussion oder Einwänden bieten, etwa bei Konzepten zum Umgang und der Zusammenarbeit mit - sowie der versuchten Instrumentalisierung von - Medien und Nichtregierungsorganisationen (NGO) oder bei der Ausfüllung von Praktiken der psychologischen Kriegführung.

Hier ist nicht der Ort, solche Fragen ausführlich zu erörtern. Es muss aber darauf hingewiesen werden, dass die politischen Kerne der Konzepte von „Military Operations Other than War“ heute vor allem in der Bekämpfung und Förderung von Aufständen bzw. dem Umgang mit failed states liegen. Genau in diesen Fragen bestehen potenzielle Meinungsunterschiede nicht allein zu wichtigen Strömungen der europäischen Öffentlichkeit, sondern auch zu großen Teilen europäischer NATO-Streitkräfte und europäischer Regierungen. So wirft die Unterstützung oder Organisierung fremder Aufständischer in der Regel schwerwiegende völkerrechtliche Probleme auf, da es sich schon definitionsgemäß um eine bewaffnete Einmischung in die inneren Angelegenheiten eines fremden Landes handelt. Und wenn wir an die US-Unterstützung etwa der nikaraguanischen Contras oder der afghanischen Mudschahedin in den 1980er Jahren denken, zeigt sich auch, dass diese entweder höchst umstritten oder von zweifelhaftem Wert waren. Schließlich haben viele der von den USA unterstützten „arabischen Afghanen“ nach ihrer Rückkehr in ihre Heimatländer dort wesentlich zur Destabilisierung der eigenen Länder beigetragen und wurden nicht selten zum Kern terroristischer Organisationen. Auch über den Umgang mit festgenommenen Terrorverdächtigen oder Aufständischen - Stichwort Guantanamo - bestehen bei verschiedenen NATORegierungen bekanntlich Meinungsverschiedenheiten. ${ }^{3}$

\section{Das Konzept der Counterinsurgency und seine Defizite}

Von besonderer Bedeutung bei militärischen Operationen unterhalb der Schwelle eines konventionellen Krieges sind heute und zukünftig Situationen von Counterinsurgency, also Aufstandsbekämpfung. Nach der offiziellen Definition des US-Militärs handelt es sich um „diejenigen militärischen, paramilitärischen, politischen, wirtschaftlichen, psychologischen und civic action ${ }^{4}$ Maßnahmen einer Regierung, die die Niederlage eines Aufstandes bewirken sollen."5

\footnotetext{
3 Siehe Hippler (2006) mit weiteren Nachweisen.

4 Civic action: Nichtmilitärische Aktivitäten des Militärs, die auf die Zivilbevölkerung zielen und dieser nutzen sollen, z.B. medizinischer Art.

5 US Department of Defense 2001: Dictionary of Military and Associated Terms, Joint Publication (JP) 1-02, 12 April (As Amended Through 8 August 2006); p.
} 127; Übersetzung hier und in allen anderen Fällen durch den Autor. 
Dazu gehören vor allem a) zivil-militärische Operationen, die wiederum Maßnahmen zum Nutzen und zur Gewinnung der Zivilbevölkerung, psychologische Operationen, humanitäre Hilfe und die Unterstützung für zivile Behörden beinhalten; b) Kampfeinsätze, vor allem gegen Guerillas; und c) Informationsoperationen. ${ }^{6}$

Das US-Militär nimmt an, dass Aufstände nur dann in Gang kommen, wenn die Bevölkerung (aus sehr unterschiedlichen Gründen) mit dem wirtschaftlichen und/oder politischen Status quo grundlegend unzufrieden sowie eine glaubwürdige, regimefeindliche politische Führungsgruppe vorhanden ist und die Regierung zumindest zum Teil die Kontrolle über die Situation verloren hat. Insgesamt seien Situationen von Aufständen und Aufstandsbekämpfung nur zum Teil militärisch, vor allem aber politisch geprägt, sie könnten daher letztlich auch nur politisch gewonnen werden. In den Worten der US-Army:

„Der Erfolg in Counterinsurgency-Operationen fällt derjenigen Seite zu, die eine größere Unterstützung durch die Bevölkerung erreicht. Diejenige Konfliktpartei wird sich durchsetzen, die die politischen Fragen besser prägt, die Gruppen und Kräfte um sich herum stärker mobilisiert und Programme entwickelt, die das Problem der relativen Deprivation (Entzug von Rechten oder Ressourcen; JH) besser löst. Dies erfordert politische, wirtschaftliche und soziale Entwicklung. "7

Die Counterinsurgency-Konzepte des US-Militärs weisen häufig darauf hin, dass solche notwendigen Reformen allerdings nicht das Ziel der Politik, sondern nur Mittel seien. Letztlich gehe es darum, in den Augen der Bevölkerung als die legitimere Konfliktpartei zu erscheinen; Ziel sei es, den „Krieg um die Legitimität“ zu gewinnen. Auch dies sei wiederum kein Selbstzweck. Letztendlich besteht die Strategie darin, die Bevölkerung von den Aufständischen zu trennen - wenn möglich politisch, sonst aber auch physisch oder geographisch. Wer die Bevölkerung kontrolliere, gewinne den Krieg. Gerade dieser letzte Punkt impliziert unter schwierigen Bedingungen großes repressives Potenzial von Counterinsurgency-Strategien, wie es im Vietnamkrieg exzessiv auftrat. ${ }^{8}$

Die Entwicklung von US-Counterinsurgency-Strategien ist nicht neu. Ein erstes entsprechendes militärisches Handbuch wurde bereits 1940 vom Marine Corps erarbeitet ${ }^{9}$, als die USA bereits über ein halbes Jahrhundert an entsprechenden Erfahrungen verfügten. Die Strategieentwicklung wurde allerdings immer wieder mittelfristig zurückgeworfen, weil etwa die beiden Weltkriege und der Kalte Krieg als vordringlich betrachtet wurden. Couterinsurgency-Konzepte wurde deshalb fast immer eher widerwillig und halbherzig entwickelt und häufig nur teilweise umgesetzt. Erst aufgrund der Schwierigkeiten des US-Militärs im Irak nach 2003 wurde die Strategieentwicklung konsequent vorangetrieben und führte im Dezember 2006 zum neuen Field

6 US Department of the Army 2004, Counterinsurgency Operations, FMI 307.22, Washington, October, p. VIII.

7 US Department of the Army 2003, Field Manual FM 3-07 (formerly FM 10020), Stability Operations and Support Operations, Washington, February, p. 3-4.

8 Eine allgemeine und breite Darstellung des veränderten Charakters des Krieges, der immer mehr um und mit der Bevölkerung geführt werde, findet sich bei Rupert Smith, The Utility of Force - The Art of War in the Modern World, London 2006.

9 US Marine Corps, Small Wars Manual, US GPO, Washington 1940, online unter: www.smallwars.quantico.usmc.mil/sw_manual.asp.
Manual 3-24 „Counterinsurgency“.$^{10}$ Dieses von General David Petraeus geprägte Handbuch betont noch stärker als seine Vorgänger den grundlegend politischen Charakter von Aufstandsbekämpfung und warnt vor einer Überschätzung der militärischen Instrumentarien.

Es lässt sich insgesamt festhalten, dass die hier nur kurz anzureißenden Counterinsurgency-Konzepte oder entsprechende Einsätze in fragmentierten Gesellschaften einen höchst widersprüchlichen Charakter tragen. Einmal besteht ein offensichtliches Spannungsverhältnis zwischen ihren militärischen Komponenten (die hier kaum angesprochen werden konnten) und den politischen und reformorientierten Aspekten - häufig blockieren sich beide in der Realität gegenseitig. Zweitens bleiben politische und reformorientierte Ansätze oft blass und vage, während die militärischen spezifisch und konkret ausgefüllt werden. Drittens sind sich viele militärische Analytiker bewusst, dass die zivilen Komponenten von Counterinsurgency zwar letztlich den Konflikt entscheiden, das Militär aber gerade hier über geringe Kompetenz verfügt. Viertens besteht zusätzlich eine beträchtliche Kluft zwischen den allgemeinen Strategien und der Realität militärischen Handelns vor Ort, wo sich sicherheitspolitische Erwägungen oft gegenüber den politischen Notwendigkeiten durchsetzen. Und fünftens hat die Strategie eine konzeptionelle Achillesferse: Seine Schlüsselelemente zielen fast immer auf die Unterstützung einer lokalen Regierung - wenn eine solche aber Teil des Problems ist (durch Repression, Inkompetenz, Korruption, etc.) oder in Kontexten von failed states überhaupt nicht oder nur auf dem Papier existiert, fehlt vielen Aspekten der Counterinsurgency-Konzepte die Voraussetzung zum Erfolg. Gelegentlich wird auf dieses Problem auch in den einschlägigen Strategiepapieren hingewiesen:

„In extremen Fällen finden sich die USA und andere Länder in einer Situation, in der sie - anstatt darauf aufzubauen, was vorhanden ist - erst einmal selbst Elemente wie Streitkräfte und Regierungsbehörden in der Gesellschaft schaffen müssen, zu deren Unterstützung sie entsandt wurden. Militärische Einheiten sind dann mit Nation-Building beschäftigt, während sie zugleich versuchen, einen Aufstand niederzuschlagen."11

Eine solche Doppelaufgabe erweist sich sehr häufig als ungemein anspruchsvoll und wird in der Regel nur bei besonders günstigen innergesellschaftlichen Voraussetzungen gelingen. In Afghanistan und im Irak zeigen sich die Probleme externer Aufstandsbekämpfung in Verbindung mit Nation-Building besonders deutlich. Obwohl die USA über ausgereifte - wenn auch problematische - Strategien zur Aufstandsbekämpfung verfügen, die aufgrund der neuen Erfahrungen in beiden Ländern auch kontinuierlich verfeinert werden, gelang bisher weder eine sicherheitspolitische Stabilisierung noch die stabile Etablierung funktionierender nationalstaatlicher Strukturen. Zwar darf man nicht übersehen, dass in beiden Ländern durchaus Teilerfolge beim Nation- und State-Building erzielt wurden, aber zugleich gab es schwere Rückschläge. Sieben Jahre nach der Eroberung Afghanistans und trotz aller militärischen wie ziviler Bemühungen um Sicherheit und Staatsbildung ist die

10 US Army/US Marine Corps, Counterinsurgency Field Manual FM 3-24, Chicago 2007

11 US Department of the Army 2004, Counterinsurgency Operations, FMI 307.22, Washington, October, p. 1-10. 
Sicherheitslage zunehmend schlecht, was auch die politische Zukunft des Landes unsicher werden lässt.

Im März 2003 unterhielt die ISAF 4700 Soldaten aus 28 Ländern in Afghanistan, dazu kamen knapp 10.000 US-Soldaten, die nach Angaben des US-Verteidigungsministeriums nicht verstärkt werden sollten. Anfang April 2007 standen dann allein 24.300 US-Soldaten in Afghanistan, ein Jahr später schon 33.000.12 Damit lag die Zahl der ausländischen Soldaten (ISAF und OEF) zur Jahresmitte bei fast 65.000 - weitere Truppen verschiedener Länder wurden bereits angekündigt bzw. waren schon beschlossen. Damit hatte sich die Zahl der ausländischen Truppen in den letzten fünf Jahren weit mehr als vervierfacht - dazu kam die in dieser Zeitspanne ebenfalls massiv ausgeweitete Truppenstärke der afghanischen Streitkräfte. Trotz dieser Truppenverstärkung seit 2003 hat sich in der gleichen Zeitspanne die Sicherheitslage nicht entspannt, sondern dramatisch verschärft: „The U.S. military said earlier this year that suicide bombings were up $27 \%$ in 2007 over 2006. But their incidence is also up a horrifying 600\% over 2005; and all insurgent attacks are up $400 \%$ over 2005. The last Secretary-General's report to the Security Council reported 8,000 insurgency-related deaths in 2007 , of which at least 1,500 were civilians. Humanitarian workers are increasingly targets. The Secretary General reported the looting of 40 convoys delivering food for the WFP in 2007, 130 attacks against humanitarian programs, 40 relief workers killed and another 89 abducted." 13

Nach anderen Angaben gab es 2007 fast 9.000 bewaffnete Anschläge, rund zehnmal so viel wie $2004 .{ }^{14}$ Mitte 2008 wurde berichtet, dass die Angriffe um weitere 35-40 Prozent gegenüber dem Vorjahr zugenommen hätten. Und seit Mai 2008 musste das US-Verteidigungsministerium mehrfach melden, dass nun in Afghanistan mehr US-Soldaten sterben als im Irak - obwohl dort fast fünfmal so viele stationiert sind. Wer angesichts solcher Zahlen in einer rein militärischen Logik denkt, wird mit der zunehmenden Gewalt immer weitere Soldaten verlangen - der ISAF-Oberkommandierende General McNeill erklärte im März 2008, dass 400.000 Soldaten nötig seien, um Afghanistan wirklich zu befrieden, ${ }^{15}$ also noch 100.000 mehr, als Rudolf Scharping zu seiner Zeit als Verteidigungsminister für nötig erklärt hatte.

Dies illustriert, dass in Situationen von Counterinsurgency die Stärkung der militärischen Machtmittel und die Intensivierung militärischen Zwangs häufig von sekundärer Bedeutung sind und eher zu einer Eskalation der militärischen Gewalt denn zu einer Lösung beitragen. Dieser Tatbestand, der sich vom Vorrang politischer Lösungsansätze bei der Aufstandsbekämpfung ableitet, ist im Militär - siehe das erwähnte Field Manual 3-24 - wie in der Politik durchaus bekannt, wird aber bei der Politikimplementierung häufig ignoriert oder nur zum geringen

12 JoAnne O'Brian / Michael Waterhouse, US Forces in Afghanistan, Congressional Research Service, Washington May 9, 2008, S. 5.

13 Assessing the Overall Security Situation in Afghanistan, Speech by Nick Grono, Deputy President, International Crisis Group, DCAF - NATO Parliamentary Assembly Seminar on „Stabilising Afghanistan: Developing Security, Securing Development“, 17 April 2008, online: www.crisisgroup.org/home/ index.cfm?id=5396\&l=1.

14 Ullrich Fichtner, Afghanistan - Ein Dritter Weltkrieg, in: Spiegel, 22/2008, 26.5.2008, S. $122 \mathrm{f}$.

15 Zit. nach: Ullrich Fichtner, Afghanistan - Ein Dritter Weltkrieg, in: Spiegel, 22/2008, 26.5.2008, S. $122 \mathrm{f}$.
Teil umgesetzt. In Afghanistan beispielsweise würde erfolgreiche Counterinsurgency zuerst einmal die Etablierung funktionierender und legitimer Staatlichkeit in den Provinzen und Dörfern voraussetzen (nicht allein in Kabul und den größeren Städten). Diese zentrale Aufgabe der Schaffung bürgernaher Staatlichkeit wurde allerdings weitgehend vernachlässigt und zugunsten der Stärkung sicherheitspolitischer Potenziale hintangestellt. ${ }^{16}$

Aus dem Irak wurde erst nach dem Krieg eine Brutstätte des Terrorismus, die Sicherheitslage hat sich seit 2007 zwar deutlich gebessert, bleibt aber sehr ernst. Das US-Militär traut es der irakischen Regierung weiterhin nicht zu, selbst für Sicherheit zu sorgen. ${ }^{17}$ In beiden Ländern lässt sich studieren, dass Strategien zur Aufstandsbekämpfung deren Erfolg nicht garantieren, selbst nach militärisch siegreich geführten Kriegen. In beiden Fällen fehlt es nicht an Finanzmitteln, nicht an militärischem und zivilem Personal, am politischen Willen der externen Akteure und nicht an durchdachten allgemeinen Konzepten und Strategien für Aufstandsbekämpfung. In beiden Fällen waren die externen Aufstandsbekämpfer - insbesondere die USA und ihr Militär - zu Beginn der Operationen in einer militärisch, politisch und machtpolitisch extrem starken Position, da nach den beiden Kriegen die organisierten Hauptgegner militärisch geschlagen und z.T. zerschlagen waren. Machtpolitische Alternativen zu den Besatzungstruppen gab es zu Beginn nicht. Deshalb drängt sich die Frage auf, wie trotz dieser scheinbar so günstigen Ausgangsbedingungen das Mischkonzept von Aufstandsbekämpfung und Nation-Building so große Umsetzungsschwierigkeiten haben konnte. Diese Frage ist auch deshalb bedeutsam, weil es sich hier um eine Einsatzform handelt, die in ähnlicher und anderer Form bei Operationen „Other than War" zukünftig immer wieder auftreten dürfte, da sie einen Kernbereich dessen abdeckt, was die NATO in ihrer Strategie als „Stabilisierung“ und „Sicherheit“ zum Kernbestand ihrer Aufgaben erklärt hat.

Lassen wir hier die direkten Fehler der externen Akteure (schlechte Vorbereitung etc.) einmal außer Acht, ${ }^{18}$ dann liegen die strukturellen Probleme auf zwei Ebenen:

Widersprüche und Zielkonflikte der externen Politik im Zielland. In Afghanistan widersprechen z.B. die Ziele gesellschaftlicher Stabilisierung und der Bildung eines funktionierenden Nationalstaats nicht selten den Interessen an militärischer Sicherheit und der Kriegführung gegen die Taliban und von Al-Qaida. Auch wenn sich beides vom Schreibtisch aus als vereinbar darstellen mag, blockierten sie sich doch oft gegenseitig und wurden gar institutionell in der Doppelstruktur von ISAF und der Operation Enduring Freedom reproduziert. Die gegenwärtige bürokratische Lösung einer schrittweisen organisatorischen Integration beider bei inhaltlicher Akzentverschiebung in Richtung Kampfeinsatz löst dieses Problem nicht, sondern dürfte

16 Dazu: Jochen Hippler, Afghanistan: Kurskorrektur oder Rückzug? - Die politischen Folgen aus der Gewalteskalation, Policy Paper 29 der Stiftung Entwicklung und Frieden (SEF), Bonn 2008.

17 Iraqi Cabinet Wants Security Agreement Altered, in: New York Times, October 22, 2008, online: www.nytimes.com/2008/10/22/world/middleeast/22iraq. html?sq=Iraq security\&st=cse\&scp=2\&pagewanted=print.

18 Siehe dazu Jochen Hippler, Militärische Besatzung als Schöpfungsakt - Nation-Building im Irak, in: Jochen Hippler (Hrsg.), Nation-Building - ein sinnvolles Instrument der Konfliktbearbeitung?, Bonn 2004, S. 121-140. 
mittelfristig die Probleme eher noch vertiefen. Ähnliche Zielkonflikte bestanden und bestehen im Irak, wo man zwischen dem Interesse nach imperialer Kontrolle und der militärischen Sicherung der eigenen Truppenpräsenz einerseits und dem an demokratischer Staatsbildung unter local ownership hin und her pendelt. So haben die Besatzungstruppen die Ethnisierung der irakischen Politik, die sich heute als so gewaltträchtig erweist, nach dem Krieg selbst gefördert, weil ihnen nur so die Aufgaben der Sicherheit und einer späteren Machtübergabe erreichbar schienen.

Fehlende oder schwache Partner im Zielland. Aufstandsbekämpfung und demokratisches Nation-Building können offensichtlich zwar von außen unterstützt, aber nicht von externen Akteuren getragen werden. Die US-amerikanischen Counterinsurgency-Experten sind sich dieser Problematik zwar im Kern bewusst, was aber nichts daran ändert, dass eine tragfähige soziale Basis für entsprechende Politik vor Ort oft fehlt. In Afghanistan war und ist nicht erkennbar, welche relevanten Sektoren der afghanischen Gesellschaft eigentlich einen demokratischen Staatsbildungsprozess tragen sollen, der von außen unterstützt werden könnte: Die lokalen Warlords kommen ebenso wenig in Frage wir die frühere Nordallianz, die Taliban oder die Mehrheit der Landbevölkerung, die weiter in extrem konservativen und patriarchalischen lokalen Machtstrukturen gefangen ist. Eine zahlenmäßig starke, wirtschaftlich und politisch selbstbewusste Mittelschicht - eine potenzielle Basis für demokratische Staatsbildung - existiert weiterhin nicht, so dass die Staatsbildung im Land nur durch die Staatsbeamten selbst und eine sehr kleine Schicht gebildeter Exilrückkehrer getragen wird. Das ist erkennbar zu wenig, um einen von innen gestützten, selbsttragenden Demokratisierungsschub zu entwickeln. Auch im Irak sieht es nur wenig besser aus. Dort können sich die USA vor allem auf die beiden säkularen kurdischen Parteien stützen, während sie im Rest des Landes inzwischen im besten Fall als notwendiges Übel toleriert werden. Und die kurdischen Parteien haben nur ein taktisches Interesse an irakischer Staatsbildung, da sie nach dem Krieg vor allem an einer kurdischen Eigenstaatlichkeit interessiert sind.

\section{Die Notwendigkeit alternativer Strategien für die NATO}

Die NATO und ihre Mitgliedsländer haben durch ihre Strategie von 1999 und ihre Einsätze seit dem Beginn der 1990er Jahre (insbesondere aber seit Beginn des Afghanistan-Einsatzes) die Tür zu unkonventionellen Einsatzformen geöffnet, die in sehr unterschiedlichen Kontexten und unter sehr unterschiedlichen Bezeichnungen Elemente von Counterinsurgeny und NationBuilding enthalten. Dies gilt für uni- wie multilaterale Einsätze und solche im organisatorischen Rahmen der NATO.

Nicht alle der gegenwärtigen und zukünftigen Operationen werden so dramatisch verlaufen wie die aktuellen im Irak und Afghanistan, aber sie werden zahlreichen der dort zu beobachtenden Probleme in anderer, milderer oder ähnlicher Form wieder begegnen. Sollten sich die NATO oder ihre Mitgliedsländer weiter in entsprechende Situationen begeben, ohne dass die grundlegenden Voraussetzungen für einen Erfolg bestehen (kohärente Politikziele, eine seriöse politische Gesamtstrategie und vor allen eine tragfähige soziale Basis im Zielland) dürften solche Operationen langfristig Konflikte eher verschärfen und das Gewaltniveau der betroffenen Länder noch heben. ${ }^{19}$ Das liegt weder im Interesse dieser, noch in der Regel der der intervenierenden Länder. Deshalb ist es von hoher Bedeutung, dass die NATO-Mitgliedstaaten, soweit sie nicht imperiale Interessen verfolgen, sondern an konstruktiver Konfliktbearbeitung interessiert sind, erstens selbst Strategien und Konzepte entwickeln, die kohärent, wirksam und konfliktbearbeitend sind, anstatt schrittweise US-amerikanischen Counterinsurgency-Strategien zu übernehmen. Dabei lässt sich an solche Konzepte durchaus punktuell anknüpfen. Es käme aber darauf an, ihre politischen und sozioökonomischen Lösungsansätze tatsächlich ins Zentrum der eigenen Politik zu rücken, anstatt sie zu bloßen Instrumenten militärischer Operationen werden zu lassen. Zweitens müssen die NATO-Länder vor entsprechenden Einsätzen ernsthaft und systematisch prüfen, ob im Zielland der Operation überhaupt die grundlegenden inneren Voraussetzungen für einen Erfolg bestehen. Wo sie fehlen, sind Truppeneinsätze nicht verantwortbar, weder für ein fragiles Zielland, noch für die Soldaten. ${ }^{20}$

Die NATO ist Ende der 1990er Jahre der Versuchung erlegen, ihre Existenzberechtigung mit einem bunten Katalog von möglichen und wünschbaren Zielen und Absichten nachweisen zu wollen. Eine „Strategie“ ist so etwas nicht. Eine solche würde gerade darin bestehen, die Ziele stärker zu gewichten, sie mit den Mitteln in eine konkrete Beziehung zu setzen und zu formulieren, wie man unter Einsatz der Mittel die Ziele zu erreichen gedenkt. Davon ist bisher kaum die Rede. Nun stehen die NATO und viele Mitgliedsländer vor der Wahl, entweder durch wohlklingende Formulierungen den Mangel weiter zu verbergen, anderswo fertige Konzepte und Strategien von der Stange zu übernehmen oder sich endlich der Mühe zu unterziehen, für die laufenden und zukünftigen Einsätze seriöse und kreative konzeptionelle Arbeit zu leisten. Afghanistan ist heute die Nagelprobe für die Fähigkeit der Allianz - und der Streitkräfte ihrer wichtigsten Mitgliedsländer -, sich an die Bedingungen der neuen Einsatzformen zivil-militärischer Interventionen konzeptionell anzupassen. Eine zweite Herausforderung westlicher Friedens- und Sicherheitspolitik besteht gegenwärtig darin zu lernen, dass Zurückhaltung und Selbstbeschränkung bei militärischen Einsätzen häufig klüger sind, als mit geringer konzeptioneller Klarheit ihre militärische Übermacht dort zum Tragen bringen zu wollen, wo Gewaltkonflikte vor allem politische, ökonomische und gesellschaftliche Reformen erfordern.

19 Jochen Hippler, Bedingungen, Kriterien und Grenzen militärischer Interventionen, in: Bruno Schoch, Andreas Heinemann-Grüder, Jochen Hippler, Markus Weingardt und Reinhard Mutz (Hrsg.); Friedensgutachten 2007, Münster 2007, S. 110-121.

20 Ebenda. 